\title{
Portage du virus de l'hépatite $B$ chez les travailleuses du sexe dans trois villes secondaires du Burkina Faso: Koudougou, Ouahigouya et Tenkodogo
}

\section{Hepatitis B virus carriage among Female Sex Workers in three secondary cities of Burkina Faso: Koudougou, Ouahigouya and Tenkodogo}

Ouedraogo $\mathrm{HG}^{1,2,3}$, Nikiema $\mathrm{AR}^{1}$,Cisse $\mathrm{K}^{1}$, Ky-Zerbo $\mathrm{O}^{4}$, Samadoulougou $\mathrm{BC}^{3}$, Ouedraogo $\mathrm{AM}^{1}$, Traore $\mathrm{Y}^{2}$, Ouedraogo/Traore $\mathrm{R}^{2}$, Barro $\mathrm{N}^{2}$, Kouanda $\mathrm{S}^{1,3}$.

${ }^{1}$ Institut de Recherche en Sciences de la Santé (IRSS/CNRST), Ouagadougou, Burkina Faso ;

${ }^{2}$ Université Ouaga I Professeur Joseph KI-ZERBO, Ouagadougou, Burkina Faso;

${ }^{3}$ Institut Africain de Santé Publique (IASP), Ouagadougou ;

${ }^{4}$ Programme d'Appui au Monde Associatif et Communautaire (PAMAC), Ouagadougou, Burkina Faso.

Corresponding author: Henri Gautier Ouedraogo,: Laboratoire de recherche Biomédicale, Institut de Recherche en Sciences de la Santé (IRSS/CNRST), Ouagadougou, Burkina Faso. Email: gouedraogo@irss.bf

Résumé

Objectif : Les travailleuses du sexe (TS) sont un groupe à haut risque pour les infections sexuellement transmissibles dont le virus de l'hépatite B. L'objectif de cette étude est d'évaluer la prévalence du virus de l'hépatite B et les facteurs qui y sont associés chez les TS dans trois villes secondaires du Burkina Faso. Matériels et méthodes : Il s'est agi d'une étude transversale dans les localités de Koudougou, Ouahigouya et Tenkodogo, trois villes secondaires du Burkina Faso. Les TS ont été recrutées de février à mai 2014 par la méthode d'échantillonnage basé sur le répondant dans le cadre d'une étude de séroprévalence du VIH. Les échantillons de sérum archivés ont été testés à la recherche de l'antigène HBs. Les données ont été analysées en utilisant le logiciel STATA version 14.0. Résultats : Au total, 320 échantillons sériques de TS ont été testés à la recherche de l'antigène HBs. Les prévalences ajustées de l'Ag HBs étaient de 6,4\% (95\% IC : 3,4-11,8\%) à Koudougou, 15,7\% (95\%IC : 09,5-25,0\%) à Ouahigouya et 11,0\% (95\%IC : 6,8-17,2\%) à Tenkodogo. Les facteurs associés à l'infection étaient l'âge élevé de l'enquêtée, le nombre élevé de clients par semaine, le statut positif au VIH, la non utilisation systématique du condom. Conclusion : La prévalence de l'hépatite B et les facteurs associés dans cette étude suggèrent la nécessité de sensibiliser davantage à l'utilisation systématique du préservatif et à la vaccination contre le VHB chez les populations à haut risque, telles que les professionnelles du sexe.

Mots clés : VHB, Ag HBs, travailleuses du sexe, Burkina Faso.

\section{Summary}

Objective: Female sex workers (FSW) are one of most-at-risk population for Sexually Transmitted Infections, including Hepatitis B Virus. This study aimed to estimate the prevalence of hepatitis B virus infection and factors associated among FSW in Koudougou, Ouahigouya and Tenkodogo, three secondary towns of Burkina Faso.Methods: This was a cross-sectional study in the cities of Koudougou, Ouahigouya and Tenkodogo, three secondary towns of Burkina Faso. Female sex workers (FSWs) were recruited from February to May 2014 using respondent driven sampling (RDS) method during an HIV seroprevalence study. Participant's archived sera were tested for HBs antigen. The data was analyzed using STATA version 14.0 software. Results: A total of 320 serum samples from FSWs were tested for HBs antigen. The adjusted prevalence's of HBsAg were $6.4 \%$ (95\% CI: 3.4-11.8\%) in Koudougou, 15.7\% (95\% CI: 09.5-25.0\%) in Ouahigouya and 11.0\% (95\% CI: 6.8$17.2 \%$ ) in Tenkodogo.Factors associated with hepatitis B virus carriage were the high age of the respondent, the high number of clients per week, the positive HIV status, and the inconsistence use of condom. Conclusion: The prevalence of hepatitis B and its associated factors in this study suggest the need to increase awareness regarding consistence use condom and vaccination against HBV for high risk population, like sex workers.

Keywords: HBV, HBsAg, Female sex workers, Burkina Faso.

\section{$\underline{\text { Introduction }}$}

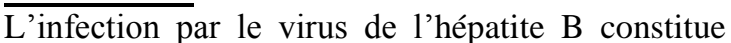
une des infections virales humaines les plus répandues au monde. Il s'agit d'une infection hépatique causée par le virus de l'hépatite B (VHB), potentiellement mortelle, très contagieuse avec pour seul réservoir l'homme $(1,2)$. Sa transmission chez l'adulte se fait principalement à travers les rapports sexuels non protégés ou le contact des muqueuses avec tout liquide biologique infecté (3). Elle représente un problème majeur de santé publique au niveau mondial (4). Selon l'Organisation Mondiale de la Santé (OMS), plus de 2 milliards d'individus ont déjà été en contact avec le VHB et 240 millions en sont porteurs chroniques. La majorité de ces cas sont en Asie et en Afrique. Le nombre annuel de décès dans le monde liés au VHB est estimé à plus de 780000 de suite cirrhose ou de cancer du foie (3). La morbidité et la mortalité liées au VHB en Afrique 
restent cependant peu connues. Les raisons de cette méconnaissance sont entre autres le manque d'un système fiable de collecte des données et la rareté des études épidémiologiques sur cette infection dans la plupart des pays africains (5). Toutefois, les estimations faites par l'OMS montrent que l'Afrique subsaharienne constitue une zone de forte prévalence avec un taux de portage chronique du VHB de plus de $8 \%$, contre moins de $1 \%$ en Europe occidentale et en Amérique du Nord $(3,5)$. Certains auteurs estiment que 70 et $95 \%$ de la population adulte africaine a déjà été en contact avec le VHB et entre 6 et $20 \%$ des Africains en sont porteurs chroniques pouvant développer plus tard des cancers ou des cirrhoses du foie (6). Les plus fortes prévalences du VHB sont observées en Afrique de l'Ouest avec notamment $17 \%$ au Sénégal (7). La forte endémicité du VHB en Afrique s'accompagne de grandes disparités entre pays, mais aussi entre les groupes étudiés à l'intérieur d'un même pays (8). Dans bon nombre de pays, les travailleuses du sexe (TS) sont considérées comme groupe à haut risque pour les infections transmises sexuellement $(9,10)$. Toutefois, les études portant sur l'épidémiologie du VHB chez les travailleuses du sexe sont rares en Afrique (11-13). C'est pour combler ce gap, que nous avons conduit cette étude dont l'objectif est d'évaluer la séroprévalence du virus de l'hépatite B ainsi que les facteurs associés chez les travailleuses du sexe de trois villes secondaires au Burkina Faso.

\section{Matériels et méthodes}

\section{Type et période d'étude}

Il s'agit d'une étude transversale utilisant les spécimens biologiques et les données comportementales collectées de février à mai 2014 chez les travailleuses du sexe dans trois villes secondaires du Burkina Faso. Les détails de la méthodologie de l'étude ont été présentés dans une de nos précédentes antérieures (14).

\section{Population d'étude et taille d'échantillon}

La population d'étude est constituée des travailleuses du sexe âgées d'au moins 18 ans dans les trois localités de l'étude. Il s'agit de Koudougou, Ouahigouya et Tenkodogo, respectivement situées dans les régions du CentreOuest, du Nord et du Centre-Est. Ces TS ont été initialement recrutées dans le cadre d'une étude de séroprévalence du VIH dans ces villes, et les échantillons de sérum ont été archivés et utilisés dans la présente étude. Au total 320 échantillons de sérum en raison de 110 à Koudougou, 89 à Ouahigouya et 121 à Tenkodogo ont été analysés à la recherche de l'antigène HBs.

Echantillonnage et recrutement des
participantes

Les travailleuses du sexe (TS) ont été recrutées selon la méthode d'échantillonnage basé sur le répondant ou «Respondent-Driven Sampling, RDS » dans chacune des villes. Le RDS est une technique d'échantillonnage probabiliste conçue pour obtenir des échantillons de populations cachées et difficiles d'accès comme les travailleuses du sexe (15). De façon pratique, il a été d'abord constitué une liste des leaders (8 à 10) de la communauté TS pour participer à l'étude en tant que premières participantes (Graines). Après avoir complété le processus de l'étude comportant l'administration d'un questionnaire sociocomportemental, et un conseil dépistage volontaire $\mathrm{du}$ VIH suivi d'un prélèvement sanguin, la «graine » a été ensuite formée pour recruter ses paires (TS). Celle-ci une fois recrutée, reçoit un maximum de trois « coupons ou cartes d'invitation » pour recruter d'autres TS dans son réseau personnel (le nombre maximum de trois étant fonction de la capacité de recrutement de l'individu), selon les critères d'inclusion de l'étude. Les recrutées qui arrivent sur le site de l'étude ont été incluses dans l'étude, et formées au recrutement avant de recevoir à leur tour un maximum de trois coupons pour recruter d'autres paires, et ainsi de suite jusqu'à l'atteinte de l'échantillon voulu dans chaque localité. Un numéro unique a été pré imprimé sur chaque coupon permettant de faire le lien entre les participants de l'étude (lien entre recruteurs et recrutés).

\section{Collecte des données comportementales}

Les travailleuses de sexe invitées à participer à l'étude ont été accueillies dans un local, réservé aux entretiens, dans lequel les critères d'éligibilité ont été vérifiés suivi d'une demande de consentement informé et signé. A l'issue de la signature du consentement, un questionnaire comportemental a été administré en face-à-face par un agent de collecte des données. Un prélèvement sanguin veineux a été effectué au pli du coude après l'administration du questionnaire pour la recherche du VIH et de la syphilis sur place et la recherche ultérieure d'autres infections sexuellement transmissibles comme l'hépatite B.

\section{Méthodes de laboratoire}

Les prélèvements sanguins ont été centrifugés à 3000 tours/minute pendant 10 minutes. Le sérum reconditionné dans deux aliquots de $1,5 \mathrm{ml}$ anonymes et conservé à $-20^{\circ} \mathrm{C}$ pour la recherche ultérieure des antigènes HBs. Les échantillons congelés ont été décongelés à température ambiante avant les analyses au laboratoire. L'Antigène HBs, marqueur d'infection au virus de l'hépatite $\mathrm{B}$ a été recherché à l'aide des tests rapides de dépistage Alere Determine ${ }^{\mathrm{TM}} \quad H B s A$ (https://www.alere.com/en/home/productdetails/determine-hbsag.html,). Il s'agit d'un test immunochromatographique pour la détection qualitative in vitro de l'antigène de surface du virus de l'hépatite B (AgHBs). Ce test approuvé par l'OMS a été utilisé avec satisfaction dans plusieurs études de séroprévalence [16-18].

Analyse des données 
Les données ont été analysées à l'aide du logiciel Stata version 14.0. La variable dépendante pour l'analyse est le portage de l'Ag HBs, variable dichotomique (Oui/Non). Les statistiques descriptives (proportion et moyenne) ont été utilisées pour la description des caractéristiques sociodémographiques des participantes (âge, situation matrimoniale, ancienneté dans le travail du sexe, etc.), la prévalence de l'Antigène HBs et le statut vaccinal anti-VHB des participantes, les comportements sexuels, l'utilisation du préservatif. Ces analyses brutes ont permi de fournir les proportions échantillonnnales (\% RDS non ajustés). Des analyses ajustées à la méthode RDS ont été faites afin d'avoir des pourcentages populationnels avec leurs intervalles de confiance (IC) à 95\% (\% RDS ajusté) en prennant en compte la probabilité de récrutement des participantes dans l'étude. Cette probabilité a été mésurée indirectement par la taille du réseau personnel donnée par chaque participante à l'étude. Des analyses univariées (Odds Ratio non ajustés) et multivariées (Odds Ratio ajustés) par regression logistique simple ont été faites pour identifier les facteurs associés à l'infection par le VHB, avec un seuil de signification de 5\%. Le seuil de signification retenu pour l'association entre la variable dépendante (Ag $\mathrm{HBs}$ ) et les variables indépendantes en analyse univariée est de $20 \%$ pour l'inclusion dans le modèle en analyse multivariée.

Considérations éthiques La participation à l'étude comportementale et de séroprévalence du VIH, origine des échantillons biologiques a été volontaire, libre, consentante et anonyme. Les participantes ont donné un consentement additionnel, individuel pour l'utilisation ultérieure de leurs échantillons sanguins à des fins de recherche en santé. Le protocole de l'étude a été approuvé par le comité d'éthique pour la recherche en santé (CERS) du Burkina Faso.

$\underline{\text { Résultats }}$

\section{Caractéristiques des travailleuses du sexe}

La moyenne d'âge des enquêtées était de 25,9 $\pm 6,5$ ans. Selon la localité, cette moyenne d'âge était plus élevée à Koudougou $(27,6 \pm 7,5$ ans) qu'à Ouahigouya $(26.3 \pm 6.3)$ et à Tenkodogo (24.1 \pm 5.2 ans). D'une manière générale, le niveau de scolarisation des travailleuses du sexe était faible (tableau 1). Plus de $73 \%$ des travailleuses du sexe enquêtées étaient célibataires. Environ 7\% des TS ont déclaré vivre en couple. Les résultats montrent que la grande majorité des travailleuses du sexe (près de 70\%) étaient mères biologiques d'au moins un enfant et plus de $52 \%$ des TS enquêtées avaient déclaré être immigrées au Burkina Faso.

\section{Prévalence de l'antigène HBs}

La prévalence brute de l'antigène $\mathrm{HBs}$ dans notre échantillon de travailleuses du sexe est de 12,8\%. Le tableau 2 montre que selon la ville, la prévalence ajustée de VHB était de 6,4\% (95\%IC :
3,4-11,8\%) à Koudougou, 15,7\% (95\%IC : 09,5$25,0 \%)$ à Ouahigouya et $11,0 \%$ (95\% IC : 6,8$17,2 \%)$ à Tenkodogo $(\mathrm{p}=0,332)$. On note que la prévalence du VHB augmente avec l'ancienneté dans le travail du sexe surtout à Koudougou et à Ouahigouya (tableau 3). L'analyse selon le statut vaccinal contre le VHB montre qu'aucune des travailleuses du sexe ayant déclaré être vaccinées n'est porteuse de l'Ag HBs, contre 6,8\%, 11,1\% et $11,1 \%$ respectivement pour les TS non vaccinées de Koudougou, Ouahigouya et Tenkodogo. Aussi, les travailleuses du sexe séropositives au VIH sont significativement plus porteuses du virus de l'hépatite B que leurs paires séronégatives, et cette disparité est effective dans les trois localités enquêtées.

Bien que la différence ne soit pas statistiquement significative au seuil de $5 \%(p=0,06)$ dans notre échantillon excepté à Tenkodogo $(p=0,023)$, on constate que les travailleuses du sexe ayant déclaré utiliser systématiquement le condom au cours des 12 derniers mois sont moins infectés par le VHB que celles chez qui le recours au condom n'a pas toujours été systématique.

Facteurs associés au portage de l'Ag HBs

Il ressort de l'analyse multivariée que les facteurs associés au portage du VHB dans notre étude sont l'âge de l'enquêtée, le nombre élevé de clients, la non utilisation du préservatif et le statut sérologique VIH positif (tableau 4). En effet, les travailleuses du sexe dont le statut sérologique VIH est négatif ont un risque diminué de $69 \%$ d'être porteuses du VHB $\left(O R_{a}=0,31, P=0,019\right)$ que celles ayant un statut positif au VIH. De même, comparé à une utilisation non systématique du condom durant les douze derniers mois, l'utilisation systématique diminue de plus de $80 \%$ le risque d'infection par le VHB chez les TS $\left(O R_{a}=0,20\right.$, $p=0,019$ ). Quant au nombre de clients par semaine, l'analyse mutlivariée confirme que l'augmentation du nombre de clients par semaine augmente le risque d'être porteuse du VHB. Cette augmentation $\mathrm{du}$ risque est de $01 \%$ par client supplémentaire $\left(O R_{a}=1,01, p=0,036\right)$.

\section{Discussion}

L'épidémiologie du virus de l'hépatite B chez les travailleuses du sexe reste peu étudiée en Afrique subsaharienne. Malgré la disponibilité d'un vaccin efficace contre le virus de l'hépatite $\mathrm{B}$ depuis les années 80 (19), notre étude montre que la prévalence de l'antigène HBs, marqueur d'infection courante du VHB reste élevée au Burkina Faso. En effet, entre $6,8 \%$ et $15,7 \%$ des travailleuses du sexe des villes enquêtées étaient porteuses du virus de l'hépatite B. Ce résultat confirme la forte endémicité du virus de l'hépatite $\mathrm{B}$ dans la région subsaharienne de l'Afrique, mais surtout le risque que représente le travail du sexe dans l'infection par le VHB. Toutefois, les prévalences trouvées notre étude sont inférieures à celles récemment 
rapportée chez les TS (20) et chez les hommes qui ont des rapports sexuels avec d'autres hommes (21) dans la plus grande ville du Burkina Faso (Ouagadougou) qui étaient respectivement de $18.2 \%$ [, et de $20.4 \%$, De même au Nigéria (22), et au Brésil (23) des auteurs ont rapporté des prévalences respectives du VHB de $17,1 \%$ et $23,1 \%$ chez les travailleuses du sexe (24). Plusieurs facteurs pris isolement en analyse uni variée sont associés au portage du virus de l'hépatite B dans notre étude. Ces facteurs sont entre autres l'âge de l'enquêtée et son ancienneté dans le travail du sexe. D'autres auteurs avaient déjà rapporté une association entre l'âge et l'infection par le VHB $(22,23)$, tout comme l'association avec l'ancienneté dans le travail du sexe (23). Cela est d'autant justifié que plus longtemps que dure l'activité du commerce du sexe, plus l'accumulation du risque est grande pour contracter les infections sexuellement transmissibles dont le VHB, surtout lorsque l'utilisation $\mathrm{du}$ condom n'est pas systématique.La non utilisation systématique du condom, est significativement associée au risque d'être infectée par le VHB dans notre étude. Cette non utilisation pourrait s'expliquer en partie par la difficulté qu'éprouvent certaines TS à négocier le port du condom avec les clients (25). Dans notre étude environ $13,7 \%$ des TS ont déclaré avoir toujours des difficultés à négocier le condom avec les clients. Cette difficulté à négocier le port du condom avec les clients a aussi été rapportée dans d'autres études en Afrique, et serait liée aux clients. A Madagascar par exemple, selon une étude auprès des TS, 32,2\% des clients refusent le port du condom et $21 \%$ réagissent violemment lorsque ceci leur est demandé. Des constats similaires sont faits au Ghana, en Gambie et en Uganda, où les raisons de la non utilisation du condoms chez les TS étaient relatives aux clients qui, soit le refusaient, soit proposaient plus d'argent que la somme habituelle (24-26). Il faut aussi noter, que certaines TS sur instruction de leurs employeurs ne peuvent exiger le port du condom à certains clients (29). Aussi, l'augmentation du nombre de clients constitue un risque supplémentaire pour l'infection par le virus de l'hépatite B. Les moyens légaux de réduire le nombre de clients chez les travailleuses du sexe dans un contexte où ni le commerce du sexe, ni la fréquentation des travailleuses du sexe n'est puni par la loi, comme au Burkina Faso (10), demeurent la sensibilisation, l'éducation des populations et la promotion des bonnes meurs. A cela, il faut ajouter l'amélioration des conditions socio-économiques des TS. Dans une étude comparant le niveau du risque chez les travailleuses du sexe selon leur statut socio-économique, Lurie et collaborateurs au Brésil avaient trouvé que plus le niveau socioéconomique est faible, plus le nombre de clients est élevé, et les TS de faible niveau socio-économique étaient 2 à 3 fois plus infectées par le VIH (17\% versus 4\%), la syphilis (60\% versus $24 \%$ ) et le VHB (52\% versus $26 \%$ ) que celles ayant un niveau économique plus élevée (30). L'amélioration du niveau socio-économique des travailleuses du sexe à travers les microfinances pour des activités génératrices de revenu pourrait renforcer leur autonomie financière (44). Nos résultats montrent que les travailleuses du sexe séronégatives au VIH sont moins à risque d'être porteuses du VHB que celles séropositives. Le virus de l'hépatite $B$ partage les mêmes modes de transmission que le VIH, et l'utilisation du condom constitue un moyen efficace de prévention contre la transmission sexuelle de ces virus dans le travail du sexe. Cependant, contrairement au VIH dont l'infection évolue toujours vers la chronicité $(31,32)$, l'infection par le VHB est dans la grande majorité (90-95\%) des cas fortuitement éliminée chez l'adulte immunocompétent $(33,34)$. L'immunodépression est connue être un facteur déterminant dans l'histoire naturelle de l'infection par le virus de l'hépatite B. Des observations cliniques avaient montré que les sujets souffrant d'immunodépression étaient plus susceptibles d'évoluer vers le portage chronique du VHB lorsqu'ils sont infectés $(35,36)$. De même, plusieurs études évaluant la prévalence du VHB chez les personnes infectées par le VIH ont rapporté que les co-infections VHB/VIH étaient fréquentes [36-38] et le VIH du fait de l'immunodépression qu'il occasionne favorise la persistance du VHB et aggrave le pronostic de l'infection $(40,41)$. La vaccination contre le virus de l'hépatite $B$, de par ses preuves d'efficacité reste le moyen le plus sûr de contrôler l'incidence du VHB chez les travailleuses du sexe. Sa promotion devrait s'étendre à ces populations à risques encore très peu couvertes, à travers des stratégies et des schémas vaccinaux adaptés $(42,43)$. Dans notre étude, moins de $5 \%$ des TS enquêtées ont déclaré avoir déjà reçu au moins une dose de vaccin antiVHB, preuve que la pratique de prévention par la vaccination n'est pas encore courante dans le milieu prostitutionnel.

Cette étude pourrait comporter des limites, intrinsèques à la méthode $\operatorname{RDS}(44,45)$. Aussi, l'auto rapportage des données comportementales par les travailleuses du sexe pourrait constituer un biais du fait des possibles oublis ou du désidérata social des travailleuses du sexe qu'il est impossible de vérifier. Enfin, les tests rapides utilisés ne détectent que l'antigène $\mathrm{HBs}$, et ne permettent pas d'identifier les porteuses chroniques du virus. En dépit de ces limites, notre étude est la première à expérimenter la méthode RDS dans le contexte burkinabè et fournit des résultats épidémiologiques intéressants sur l'infection par le virus de l'hépatite B chez les travailleuses du sexe dans des localités habituellement peu couvertes par les études antérieures. 


\section{Conclusion}

Cette étude conduite chez les travailleuses du sexe montre que la prévalence du VHB dans le milieu prostitutionnel reste relativement élevée dans les trois villes de l'étude. Elle fournit des données épidémiologiques complémentaires à celles existantes en Afrique pour le plaidoyer et la planification des actions de contrôle de l'hépatite $\mathrm{B}$ notamment la vaccination contre l'hépatite $B$ pour

1. Hou J, Liu Z, Gu F. Epidemiology and Prevention of Hepatitis B Virus Infection. Int $\mathrm{J}$ Med Sci [Internet]. 2005 Jan 5 [cited 2018 May 2];2(1):50-7. Available from: https://www.ncbi.nlm.nih.gov/pmc/articles/PMC11 42225/

2. Franco E, Bagnato B, Marino MG, Meleleo C, Serino L, Zaratti L. Hepatitis B: Epidemiology and prevention in developing countries. World $\mathbf{J}$ Hepatol [Internet]. 2012 Mar 27 [cited 2018 May 13];4(3):74-80. Available from: https://www.ncbi.nlm.nih.gov/pmc/articles/PMC33 21493/

3. WHO. ,Hepatitis B, Fac sheet $\mathrm{N}^{\circ} 204$, Update July 2015 [Internet]. 2015 [cited 2015 Sep 16]. Available from: http://www.who.int/mediacentre/factsheets/fs204/e $\mathrm{n} /$

4. WHO. Global policy report on the prevention and control of viral hepatitis in WHO Member States [Internet]. Geneva; 2013 Jul p. 220. Available from: ISBN: 9789241564632

5. Global Epidemiology of Hepatitis B Virus (HBV) Infection | ------- [Internet]. [cited 2015 Nov 28]. Available from: http://najms.net/v04i01p007w/

6. WHO | Hepatitis B [Internet]. [cited 2016 Jun 16]. Available from: http://www.who.int/csr/disease/hepatitis/whocdscsr lyo20022/en/index1.html\#world

7. Epidémiologie au Sénégal [Internet]. [cited 2015 Nov 28]. Available from: http://www.hepatites.sn/le-pnlh/les-hepatites-ausenegal.html

8. Musa B, Samaila A, Femi O, Borodo M, Bussell S. Prevalence of hepatitis B virus infection in Nigeria, 2000-2013: A systematic review and meta-analysis. Niger J Clin Pract [Internet]. 2015 [cited 2015 Nov 28];18(2):163. Available from: http://www.njcponline.com/text.asp?2015/18/2/163 $/ 151035$

9. Ota E, Wariki WM, Mori R, Hori N, Shibuya K. Behavioral interventions to reduce the transmission of HIV infection among sex workers and their clients in high-income countries. In: The Cochrane Collaboration, editor. Cochrane Database of Systematic Reviews [Internet]. Chichester, UK: John Wiley \& Sons, Ltd; 2011 [cited 2016 Jul 3]. Available from: http://doi.wiley.com/10.1002/14651858.CD006045 .pub3 les populations à haut risque comme les travailleuses du sexe, tout en incluant le contrôle du statut vaccinal pour une meilleure protection. En outre, elle suggère le renforcement de la sensibilisation pour une utilisation systématique du condom chez les travailleuses du sexe

Conflit d'intérêt : aucun.

Références

10. Ngugi EN, Roth E, Mastin T, Nderitu MG, Yasmin S. Female sex workers in Africa: Epidemiology overview, data gaps, ways forward. SAHARA-J J Soc Asp HIVAIDS [Internet]. 2012 Sep [cited 2016 Jul 4];9(3):148-53. Available from:

http://www.tandfonline.com/doi/abs/10.1080/1729 0376.2012.743825

11. Melhem NM, Rahhal N, Charide R, Kreidieh K, El-Khatib R. Human immunodeficiency virus and viral hepatitis among high-risk groups: Understanding the knowledge gap in the Middle East and North Africa Region. World J Hepatol [Internet]. 2015 Nov 8 [cited 2018 May 22];7(25):2619-30. Available from: https://www.wjgnet.com/1948-

5182/full/v7/i25/2619.htm

12. Niama FR, Loukabou Bongolo NC, Mayengue PI, Mboussou FF, Kombo Bayonne ES, Kouckodila Nzingoula FM, et al. A study on HIV, Syphilis, and Hepatitis B and $\mathrm{C}$ virus infections among female sex workers in the Republic of Congo. Arch Public Health [Internet]. 2017 May 8 [cited 2018 May 19];75. Available from: https://www.ncbi.nlm.nih.gov/pmc/articles/PMC54 21326/

13. Forbi JC, Onyemauwa N, Gyar SD, Oyeleye AO, Entonu P, Agwale SM. High prevalence of hepatitis B virus among female sex workers in Nigeria. Rev Inst Med Trop Sao Paulo. 2008 Aug;50(4):219-21.

14. Ouedraogo HG, Ky-Zerbo O, Baguiya A, Grosso A, Goodman S, Samadoulougou BC, et al. HIV among Female Sex Workers in Five Cities in Burkina Faso: A Cross-Sectional Baseline Survey to Inform HIV/AIDS Programs. AIDS Res Treat. 2017;2017:9580548.

15. Heckathorn Douglas D. Respondent-Driven Sampling: A New Approach to the Study of Hidden Populations. Univ Calif Press Behalf Soc Study Soc Probl. 1997 May;44(2):174-99.

16. WHO. Hepatitis B surface antigen assays: operational characteristics (phase I) [Internet]. Geneva; 2001 May [cited 2015 Nov 29] p. 51. Report No.: WHO/BCT/BTS/01.4. Available from: WHO/BCT/BTS/01.4

17. Shivkumar S, Peeling R, Jafari Y, Joseph L, Pai NP. Rapid Point-of-Care First-Line Screening Tests for Hepatitis B Infection: A Meta-Analysis of Diagnostic Accuracy (1980-2010). Am J Gastroenterol [Internet]. 2012 Sep [cited 2015 Nov 
29];107(9):1306-13. Available from: http://www.nature.com/doifinder/10.1038/ajg.2012. 141

18. Franzeck FC, Ngwale R, Msongole B, Hamisi M, Abdul O, Henning L, et al. Viral Hepatitis and Rapid Diagnostic Test Based Screening for HBsAg in HIV-infected Patients in Rural Tanzania. PLoS ONE [Internet]. 2013 Mar 1 [cited 2015 Nov 29];8(3). Available from: http://www.ncbi.nlm.nih.gov/pmc/articles/PMC358 5939/

19. Meireles LC, Marinho RT, Van Damme P. Three decades of hepatitis B control with vaccination. World J Hepatol. 2015 Aug 28;7(18):2127-32.

20. Ouedraogo HG, Kouanda S, Goodman S, Lanou HB, Ky-Zerbo O, Samadoulougou BC, et al. Hepatitis B, C and Delta Viruses' Infections and Correlate Factors Among Female Sex Workers in Burkina Faso, West-Africa. Open Virol J [Internet]. 2019 Jan 28 [cited 2019 Mar 28];13(1). Available from:

https://benthamopen.com/FULLTEXT/TOVJ-13-9

21. Ouedraogo HG, Kouanda S, Grosso A, Compaoré R, Camara M, Dabire C, et al. Hepatitis $\mathrm{B}, \mathrm{C}$, and $\mathrm{D}$ virus and human $\mathrm{T}$-cell leukemia virus types 1 and 2 infections and correlates among men who have sex with men in Ouagadougou, Burkina Faso. Virol J. 2018 29;15(1):194.

22. Forbi JC, Onyemauwa N, Gyar SD, Oyeleye AO, Entonu P, Agwale SM. High prevalence of 0121.2012 .748863

27. Grosso AL, Lei EL, Ketende SC, Peitzmeier S, Mason K, Ceesay N, et al. Correlates of condom use among female sex workers in The Gambia: results of a cross-sectional survey. PeerJ [Internet]. 2015 Aug 6 [cited 2016 Jul 3];3:e1076. Available from: https://peerj.com/articles/1076

28. Adu-Oppong A, Grimes RM, Ross MW, Risser J, Kessie G. Social and Behavioral Determinants of Consistent Condom Use Among Female Commercial Sex Workers in Ghana. AIDS Educ Prev [Internet]. 2007 Apr [cited 2016 Jul 3];19(2):160-72. Available from: http://guilfordjournals.com/doi/abs/10.1521/aeap.2 007.19.2.160

29. Aklilu M, Messele T, Tsegaye A, Biru T, Mariam DH, van Benthem B, et al. Factors associated with HIV-1 infection among sex workers of Addis Ababa, Ethiopia. AIDS Lond Engl. 2001 Jan 5;15(1):87-96.

30. Lurie P, Fernandes ME, Hughes V, Arevalo EI, Hudes ES, Reingold A, et al. Socioeconomic status and risk of HIV-1, syphilis and hepatitis B infection among sex workers in São Paulo State, Brazil. Instituto Adolfo Lutz Study Group. AIDS Lond Engl. 1995 Jul;9 Suppl 1:S31-37.

31. Jaffar S, Grant AD, Whitworth J, Smith PG, Whittle H. The natural history of HIV-1 and HIV-2 infections in adults in Africa: a literature review. hepatitis B virus among female sex workers in Nigeria. Rev Inst Med Trop São Paulo. 2008 Aug;50(4):219-21.

23. Schuelter-Trevisol F, Custódio G, Silva ACB da, Oliveira MB de, Wolfart A, Trevisol DJ. HIV, hepatitis B and C, and syphilis prevalence and coinfection among sex workers in Southern Brazil. Rev Soc Bras Med Trop. 2013 Aug;46(4):493-7.

24. Todd CS, Nasir A, Stanekzai MR, Bautista CT, Botros BA, Scott PT, et al. HIV, hepatitis B, and hepatitis $\mathrm{C}$ prevalence and associated risk behaviors among female sex workers in three Afghan cities. AIDS Lond Engl [Internet]. $2010 \mathrm{Jul}$ [cited 2016 Jun 12];24(0 2):S69-75. Available from:

http://www.ncbi.nlm.nih.gov/pmc/articles/PMC365 0731/

25. Chersich MF, Luchters S, Ntaganira I, Gerbase A, Lo Y-R, Scorgie F, et al. Priority interventions to reduce HIV transmission in sex work settings in sub-Saharan Africa and delivery of these services. J Int AIDS Soc [Internet]. 2013 Mar 4 [cited 2016 Jul 3];16(1). Available from: http://www.jiasociety.org/index.php/jias/article/vie w/17980

26. Bukenya J, Vandepitte J, Kwikiriza M, Weiss HA, Hayes R, Grosskurth H. Condom use among female sex workers in Uganda. AIDS Care [Internet]. 2013 May 8 [cited 2016 Jul 3];25(6):767-74. Available from: http://www.tandfonline.com/doi/abs/10.1080/0954

Bull World Health Organ [Internet]. 2004 Jun [cited 2016 Jul 4];82(6):462-9. Available from: http://www.ncbi.nlm.nih.gov/pmc/articles/PMC262 2848/

32. Sabin CA, Lundgren JD. The natural history of HIV infection: Curr Opin HIV AIDS [Internet]. 2013 May [cited 2016 Jul 4];1. Available from: http://content.wkhealth.com/linkback/openurl?sid= WKPTLP:landingpage \&an=01222929-90000000099677

33. Pan CQ, Zhang JX. Natural History and Clinical Consequences of Hepatitis B Virus Infection. Int J Med Sci [Internet]. 2005 Jan 5 [cited 2016 Jul 4];2(1):36-40. Available from: http://www.ncbi.nlm.nih.gov/pmc/articles/PMC114 2223/

34. McMahon BJ. The natural history of chronic hepatitis B virus infection. Hepatology [Internet]. 2009 May [cited 2016 Jul 4];49(S5):S45-55. Available from: http://doi.wiley.com/10.1002/hep.22898

35. Taghavi SA, Tabibi M, Eshraghian A, Keyvani H, Eshraghian H. Prevalence and clinical significance of hepatitis B Basal core promoter and precore gene mutations in southern Iranian patients. Hepat Mon. 2010;10(4):294-7.

36. de Franchis R, Meucci G, Vecchi M, Tatarella M, Colombo M, Del Ninno E, et al. The 
natural history of asymptomatic hepatitis B surface antigen carriers. Ann Intern Med. 1993 Feb $1 ; 118(3): 191-4$.

37. Sereno L, Mesquita F, Kato M, Jacka D, Nguyen TTV, Nguyen TN. Epidemiology, responses, and way forward: the silent epidemic of viral hepatitis and HIV coinfection in Vietnam. J Int Assoc Physicians AIDS Care Chic Ill 2002. 2012 Oct;11(5):311-20.

38. Barth RE, Huijgen Q, Taljaard J, Hoepelman AIM. Hepatitis $\mathrm{B} / \mathrm{C}$ and HIV in sub-Saharan Africa: an association between highly prevalent infectious diseases. A systematic review and metaanalysis. Int J Infect Dis [Internet]. 2010 Dec [cited 2016 Jun 12];14(12):e1024-31. Available from: http://linkinghub.elsevier.com/retrieve/pii/S120197 1210024562

39. Praseeda S. D. A Study on the HBV and the HCV Infections in Female Sex Workers and their Co-Infection with HIV. J Clin Diagn Res [Internet]. 2013 [cited 2016 Jun 12]; Available from: http://www.jcdr.net/article_fulltext.asp?issn=0973709 x $\&$ year $=2013 \&$ volume $=7 \&$ issue $=2 \&$ page $=234$ \&issn=0973-709x\&id=2735

40. Ranjbar R, Davari A, Izadi M, Jonaidi N, Alavian SM. HIV/HBV Co-Infections: Epidemiology, Natural History, and Treatment: A Review Article. Iran Red Crescent Med J [Internet]. 2011 Dec [cited 2016 Jul 4];13(12):85562. Available from: http://www.ncbi.nlm.nih.gov/pmc/articles/PMC337 1904/

41. Lacombe K, Bottero J, Lemoine M, Boyd A, Girard PM. HIV/hepatitis B virus co-infection: current challenges and new strategies. J Antimicrob
Chemother [Internet]. 2010 Jan 1 [cited 2016 Jul 4];65(1):10-7. Available from: http://www.jac.oxfordjournals.org/cgi/doi/10.1093/ jac/dkp414

42. Jin H, Tan Z, Zhang X, Wang B, Zhao Y, Liu P. Comparison of Accelerated and Standard Hepatitis B Vaccination Schedules in High-Risk Healthy Adults: A Meta-Analysis of Randomized Controlled Trials. Nishiura H, editor. PLOS ONE [Internet]. 2015 Jul 21 [cited 2016 Jul 4];10(7):e0133464. Available from: http://dx.plos.org/10.1371/journal.pone.0133464

43. Van Herck K, Leuridan E, Van Damme P. Schedules for hepatitis $\mathrm{B}$ vaccination of risk groups: balancing immunogenicity and compliance. Sex Transm Infect [Internet]. 2007 Jul 11 [cited 2016 Jul 4];83(6):426-32. Available from: http://sti.bmj.com/cgi/doi/10.1136/sti.2006.022111

44. McCreesh N, Frost SDW, Seeley J, Katongole J, Tarsh MN, Ndunguse R, et al. Evaluation of Respondent-driven Sampling: Epidemiology [Internet]. 2012 Jan [cited 2016 Jun 23];23(1):138-47. Available from: http://content.wkhealth.com/linkback/openurl?sid= WKPTLP:landingpage \&an=00001648-20120100000021

45. Simic M, Johnston LG, Platt L, Baros S, Andjelkovic V, Novotny $\mathrm{T}$, et al. Exploring Barriers to 'Respondent Driven Sampling' in Sex Worker and Drug-Injecting Sex Worker Populations in Eastern Europe. J Urban Health [Internet]. 2006 Nov [cited 2016 Jun 23];83(S1):615. Available from: http://link.springer.com/10.1007/s11524-006-90986 
Tableau I: Caractéristiques sociodémographiques des travailleuses du sexe de Koudougou, Ouahigouya et Tenkodogo

Table I: socidemographic characteristics of female sex workers in Koudougou, Ouahigouya and Tenkodogo

\begin{tabular}{|c|c|c|c|c|c|c|c|}
\hline \multirow[t]{2}{*}{ Variables } & \multirow{2}{*}{$\frac{\text { Ensemble }(\boldsymbol{n}=320)}{\mathrm{n}(\%)}$} & \multicolumn{2}{|c|}{ Koudougou $(n=110)$} & \multicolumn{2}{|c|}{ Ouahigouya $(n=89)$} & \multicolumn{2}{|c|}{ Tenkodogo $(n=121)$} \\
\hline & & $\begin{array}{c}\text { n (\%RDS-non } \\
\text { ajusté) }\end{array}$ & $\begin{array}{c}\text { \% RDS-ajusté } \\
95 \% \text { IC }\end{array}$ & $\begin{array}{c}\text { n (\%RDS-non } \\
\text { ajusté) }\end{array}$ & $\begin{array}{c}\% \text { RDS-ajusté } 95 \% \\
\text { IC }\end{array}$ & $\begin{array}{c}\text { n (\%RDS-non } \\
\text { ajusté) }\end{array}$ & \% RDS-ajusté 95\% IC \\
\hline Age (ans) & & & & & & & \\
\hline $18-24$ & $165(51,6)$ & $44(40,0)$ & $40,5(31,7-50,0)$ & $45(50,6)$ & $51,7(41,3-62,0)$ & $76(62,8)$ & $63,1(54,1-71,3)$ \\
\hline $25-29$ & $88(27,5)$ & $35(31,8)$ & $31,9(23,8-41,2)$ & $24(27,0)$ & $26,0(18,0-36,0)$ & $29(24,0)$ & $23,8(17,0-32,3)$ \\
\hline Plus de 30 & $67(20,9)$ & $31(28,2)$ & $27,6(20,1-36,7)$ & $20(22,5)$ & $22,3(14,8-32,1)$ & $16(13,2)$ & $13,1(08,1-20,4)$ \\
\hline \multicolumn{8}{|l|}{ Scolarisation } \\
\hline aucun & $92(28,7)$ & $29(26,4)$ & $26,5(19,0-35,6)$ & $29(32,6)$ & $32,3(23,3-42,7)$ & $34(28,1)$ & $28,2(20,9-37,0)$ \\
\hline primaire & $109(34,1)$ & $44(40,0)$ & $39,8(31,1-49,3)$ & $21(23,6)$ & $23,2(15,6-33,1)$ & $44(36,4)$ & $36,1(28,0-45,0)$ \\
\hline secondaire ou plus & $119(37,2)$ & $37(33,0)$ & $33,7(25,4-43,1)$ & $39(43,8)$ & $44,5(34,5-55,0)$ & $43(35,5)$ & $35,7(27,6-44,7)$ \\
\hline \multicolumn{8}{|l|}{ statut matrimonial } \\
\hline célibataire & $234(73,1)$ & $78(70,9)$ & $70,9(61,7-78,7)$ & $65(73,0)$ & $73,8(63,7-81,9)$ & $91(75,2)$ & $75,5(67,0-82,4)$ \\
\hline vit en couple & $20(6,3)$ & $8(7,3)$ & $07,2(03,6-13,8)$ & $7(7,9)$ & $07,7(03,7-15,3)$ & $5(4,1)$ & $04,2(01,8-09,8)$ \\
\hline Divorcée/séparée/veuve & $66(20,6)$ & $24(21,8)$ & $21,9(15,1-30,6)$ & $17(19,1)$ & $18,6(11,8-28,0)$ & $25(20,7)$ & $20,2(14,0-28,4)$ \\
\hline \multicolumn{8}{|l|}{ Profession } \\
\hline Etudiante/élève & $19(5,9)$ & $6(5,5)$ & $05,6(02,5-11,9)$ & $2(2,2)$ & $02,3(00,6-08,9)$ & $11(9,1)$ & $09,2(05,1-15,9)$ \\
\hline travailleur & $131(40,9)$ & $54(49,1)$ & $48,7(39,5-58,1)$ & $36(40,4)$ & $39,9(30,2-50,4)$ & $41(33,9)$ & $34,0(26,1-43,0)$ \\
\hline sans emploi & $170(53,1)$ & $50(45,5)$ & $45,7(36,6-55,2)$ & $51(57,3)$ & $57,8(47,2-67,6)$ & $69(57,0)$ & $56,8(47,8-65,4)$ \\
\hline \multicolumn{8}{|l|}{ Nombre d'enfants biologiques } \\
\hline 00 & $102(31,9)$ & $28(25,5)$ & $25,6(18,2-34,6)$ & $25(28,1)$ & $28,5(20,0-38,9)$ & $49(40,5)$ & $40,8(32,4-49,8)$ \\
\hline 01 & $126(39,4)$ & $46(41,8)$ & $41,9(33,0-51,3)$ & $37(41,6)$ & $41,5(31,7-52,1)$ & $43(35,5)$ & $35,2(27,2-44,2)$ \\
\hline$\geq 02$ & $92(28,7)$ & $36(32,7)$ & $32,6(24,5-41,9)$ & $27(30,3)$ & $29,9(21,3-40,3)$ & $29(24,0)$ & $24,0(17,2-32,5)$ \\
\hline \multicolumn{8}{|l|}{ A immigré au Burkina Faso } \\
\hline Oui & $164(51,6)$ & $58(53,2)$ & $53,5(44,0-62,7)$ & $49(55,7)$ & $56,1(45,5-66,1)$ & $57(47,1)$ & $46,8(38,0-55,7)$ \\
\hline Non & $154(48,4)$ & $51(46,8)$ & $46,5(37,3-56,0)$ & $39(44,3)$ & $43,9(33,9-54,5)$ & $64(52,9)$ & $53,2(44,3-62,0)$ \\
\hline Total & $318(100)$ & $109(100)$ & & 88 (100) & & $121(100)$ & \\
\hline
\end{tabular}

${ }^{+}$(Côte d'Ivoire- Togo- Nigéria- etc.) 
Tableau II : Prévalence du VHB par ville selon les caractéristiques sociodémographiques des travailleuses du sexe Table II: HBV prevalence by city and by sociodemographic characteristics of female sex workers

\begin{tabular}{|c|c|c|c|c|c|c|c|}
\hline \multirow[t]{2}{*}{ Variables } & \multirow{2}{*}{$\begin{array}{c}\text { Ensemble } \\
\mathrm{n}(\%)\end{array}$} & \multicolumn{2}{|c|}{ Koudougou } & \multicolumn{2}{|c|}{ Ouahigouya } & \multicolumn{2}{|c|}{ Tenkodogo } \\
\hline & & n (\%RDS-non ajusté) & $\begin{array}{l}\text { \% RDS-ajusté } \\
\text { (95\% IC) }\end{array}$ & $\begin{array}{c}\text { n (\%RDS-non } \\
\text { ajusté) }\end{array}$ & $\begin{array}{c}\text { \% RDS-ajusté } \\
\text { (95\% IC) }\end{array}$ & $\begin{array}{c}\text { n (\%RDS-non } \\
\text { ajusté) }\end{array}$ & $\begin{array}{c}\text { \% RDS-ajusté } \\
\text { (95\% IC) }\end{array}$ \\
\hline \multicolumn{8}{|l|}{ Age (ans) } \\
\hline $18-24$ ans & $165(7,3)$ & $44(2,3)$ & $01,6(0,2-10,7)$ & $45(4,4)$ & $04,4(01,1-16,5)$ & $76(11,8)$ & $09,2(04,8-17,0)$ \\
\hline $25-29$ ans & $88(18,2)$ & $35(8,6)$ & $06,1(01,9-17,7)$ & $24(33,3)$ & $33,4(17,4-54,3)$ & $29(17,2)$ & $13,6(05,6-29,5)$ \\
\hline$\geq 30$ ans & $67(19,4)$ & $31(19,4)$ & $14,2(06,3-29,0)$ & $20(20,0)$ & $20,0(07,6-43,3)$ & $16(18,8)$ & $14,8(04,6-38,4)$ \\
\hline & $p=0.010$ & $p=0,041$ & & $p=0,007$ & & $p=0,658$ & \\
\hline \multicolumn{8}{|l|}{ Scolarisation } \\
\hline aucun & $92(13,0)$ & $29(6,9)$ & $04,9(01,2-18,0)$ & $29(20,7)$ & $20,7(09,5-39,5)$ & $34(11,8)$ & $09,1(03,4-22,5)$ \\
\hline primaire & $109(16,5)$ & $44(11,4)$ & $08,1(03,3-18,5)$ & $21(23,8)$ & $23,8(10,1-46,5)$ & $44(18,2)$ & $14,4(07,2-26,7)$ \\
\hline secondaire ou plus & $119(9,2)$ & $37(8,1)$ & $08,1(03,3-18,5)$ & $39(7,7)$ & $07,7(02,4-21,7)$ & $43(11,6)$ & $09,0(03,7-20,4)$ \\
\hline & $p=0.261$ & $p=0,785$ & & $p=0,183$ & & $p=0,615$ & \\
\hline \multicolumn{8}{|l|}{ statut matrimonial } \\
\hline célibataire & $234(10,7)$ & $78(9,0)$ & $06,4(03,0-13,0)$ & $65(10,8)$ & $10,8(05,2-21,2)$ & $91(12,1)$ & $09,4(05,2-16,4)$ \\
\hline vit en couple & $20(15,0)$ & $08(12,5)$ & $09,0(01,2-45,2)$ & $07(28,6)$ & $28,6(07,0-68,1)$ & $05(0,0)$ & --- \\
\hline Divorcée/séparée/veuve & $66(19,7)$ & $24(8,3)$ & $05,9(01,4-21,4)$ & $17(29,4)$ & $29,4(12,6-54,7)$ & $25(24,0)$ & $19,3(08,6-37,7)$ \\
\hline & $p=0,149$ & $P=0,937$ & & $p=0,112$ & & $p=0,239$ & \\
\hline \multicolumn{8}{|l|}{ Profession } \\
\hline Etudiante/élève & $19(5,3)$ & $06(0,0)$ & -- & $02(0,00)$ & -- & $11(9,1)$ & $07,0(00,9-37,8)$ \\
\hline travailleur & $131(15,3)$ & $54(13,0)$ & $09,3(04,4-18,7)$ & $36(22,2)$ & $22,2(11,4-38,9)$ & $41(12,2)$ & $09,5(03,9-21,3)$ \\
\hline sans emploi & $170(11,8)$ & $50(6,0)$ & $04,2(01,3-12,6)$ & $51(11,8)$ & $11,8(05,3-24,1)$ & $69(15,9)$ & $11,0(06,8-17,2)$ \\
\hline & $p=0,400$ & $p=0,38$ & & $p=0,349$ & & $p=0,763$ & \\
\hline Total & $320(12,8)$ & $110(9,1)$ & & $89(15,7)$ & & $121(14,0)$ & \\
\hline
\end{tabular}


Tableau III : Prévalence de l'infection par le VHB par localité selon certaines caractéristiques professionnelles des travailleuses du sexe

Table III: Prevalence of HBV infection by city and by socio-professional characteristics of female sex workers

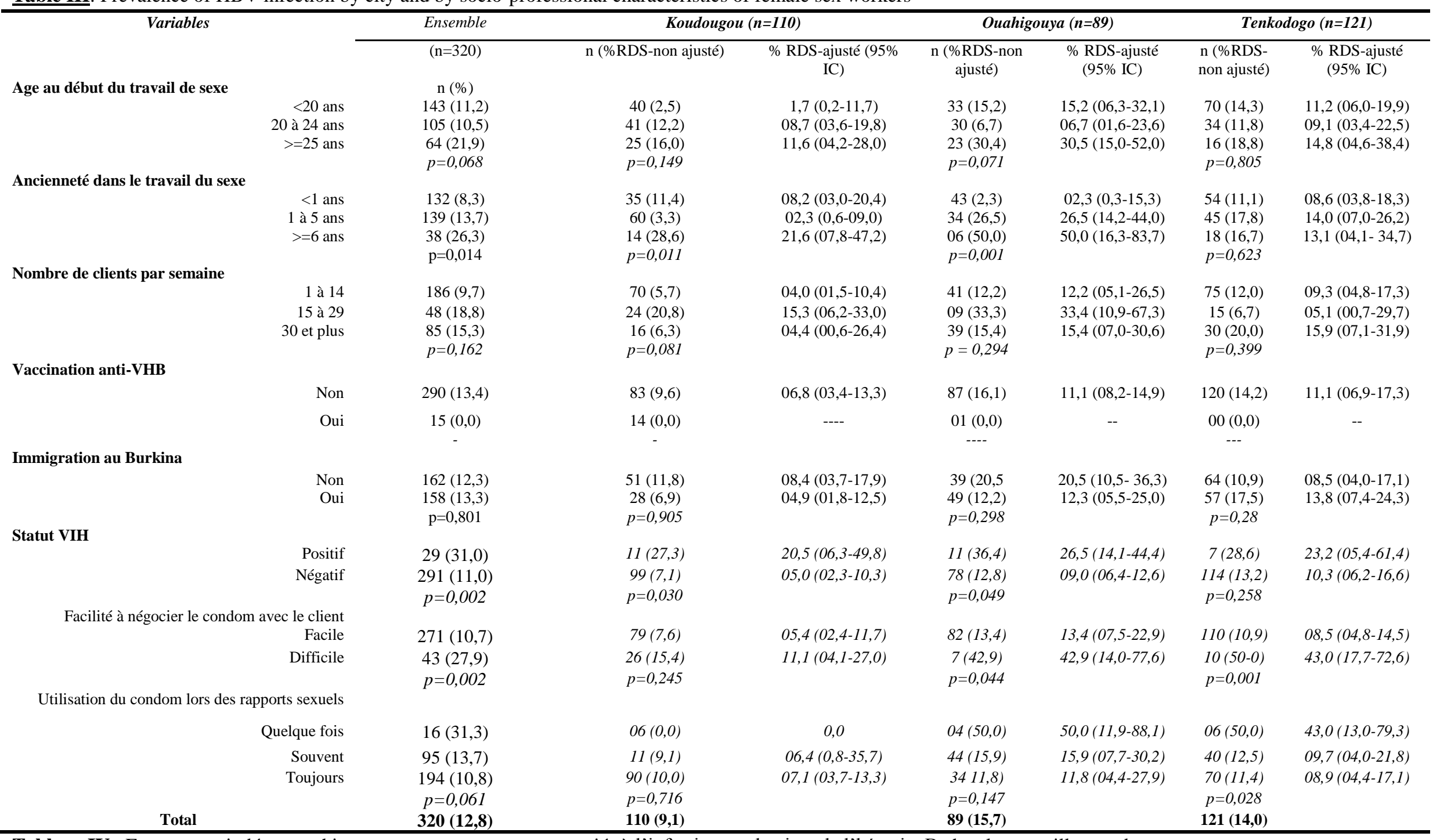

Tableau IV : Facteurs sociodémographiques et comportementaux associés à l'infection par le virus de l'hépatite B chez les travailleuses du sexe 
Table IV: Socio-demographics characteristics and behavior foctors associated to hepatitis B virus infection among female sex workers

\begin{tabular}{|c|c|c|c|c|c|c|}
\hline \multirow[t]{2}{*}{ Variables } & \multicolumn{3}{|c|}{ Analyse univariée } & \multicolumn{3}{|c|}{$\begin{array}{c}\text { Analyse multivariée } \\
\end{array}$} \\
\hline & Odds Ratio non ajusté & $(95 \% \mathrm{CI})$ & $P$ value & Odds Ratio ajusté & $(95 \% \mathrm{CI})$ & $P$ value \\
\hline Age de l'enquêté & 1,06 & $(1,01-1,10)$ & 0,007 & 1.06 & $(0.98-1.13)$ & 0.103 \\
\hline \multicolumn{7}{|l|}{ Niveau de scolarisation } \\
\hline Aucun & 1 & & & & & \\
\hline Primaire & 1,31 & $(0,60-2,90)$ & 0,492 & & & \\
\hline Secondaire et plus & 0,68 & $(0,28-1,62)$ & 0,382 & & & \\
\hline \multicolumn{7}{|l|}{ Situation matrimoniale } \\
\hline Célibataire & 1 & & & & & \\
\hline Vit en couple & 1,47 & $(0,40-5,38)$ & 0,556 & & & \\
\hline Divorcée/Séparée/veuve & 2,05 & $(0,98-4,27)$ & 0,055 & & & \\
\hline Nombre d'enfants biologiques & 1,26 & $(0,92-1,72)$ & 0,152 & 0.94 & $(0,60-1,49)$ & 0.803 \\
\hline \multicolumn{7}{|l|}{ Age au début du travail de sexe } \\
\hline$<20$ ans & 1 & & & & & \\
\hline 20 à 24 ans & 0,92 & $(0,41-2,10)$ & 0,859 & & & \\
\hline$>=25$ ans & 2,22 & $(1,01-4,89)$ & 0,047 & & & \\
\hline \multicolumn{7}{|l|}{ Ancienneté dans le travail du sexe } \\
\hline$<1$ an & 1 & & & 1 & & \\
\hline 1 à 5 ans & 1,74 & $(0,79-3,81)$ & 0,166 & 1.19 & $(0.48-2.95)$ & 0.701 \\
\hline 6 ans et plus & 3,92 & $(1,52-10,15)$ & 0,005 & 1.60 & $(0.44-5.84)$ & 0.473 \\
\hline \multicolumn{7}{|l|}{ A migré au Burkina Faso } \\
\hline Non & 1 & & & & & \\
\hline Oui & 0,88 & $(0,45-1,69)$ & 0,702 & & & \\
\hline Nombre de clients par semaine & 1,01 & $(1,00-1,02)$ & 0,039 & 1.01 & $(1.00-1.02)$ & 0.036 \\
\hline \multicolumn{7}{|l|}{ Statut sérologique négatif au VIH } \\
\hline Positif & 1 & & & 1 & & \\
\hline Négatif & 0,27 & $(\mathbf{0 , 1 1 - 0 , 6 5 )}$ & 0,004 & 0.31 & $(\mathbf{0 . 1 1 - 0 . 8 3 )}$ & 0.019 \\
\hline \multicolumn{7}{|l|}{ Négociation du port du condom avec le client } \\
\hline $\begin{array}{lll}2 & \text { Facile }\end{array}$ & 1 & & & 1 & & \\
\hline Difficile & 3,23 & $(1,49-6,97)$ & 0,003 & 3.48 & (1.44-8.39) & 0.006 \\
\hline \multicolumn{7}{|l|}{ Utilisation du condom durant les 12 derniers mois } \\
\hline Quelques fois & 1 & & & 1 & & \\
\hline Très souvent & 0,34 & $(0,10-1,16)$ & 0,088 & 0.27 & $(0.06-1.20)$ & 0.087 \\
\hline Toujours & 0,26 & $(0,08-0,84)$ & 0,024 & 0.20 & $(0.05-0.77)$ & 0.019 \\
\hline
\end{tabular}

ù 